\section{Braucht Deutschland eine Pflegekammer? Pflegeverbände fordern die Einrichtung einer Berufskammer}

In anderen Ländern gibt es schon lange eine Pflegekammer. Die Pflegeverbände in Deutschland kämpfen seit Jahren für die Einrichtung einer Berufskammer, die für die Qualität der Pflege viele Vorteile bieten würde. Bisher vergeblich, denn die Widerstände aus der Politik sind groß: Die Gegner wenden in der Regel ein, dies ginge rechtlich nicht, weil Berufskammern Selbstständigen vorbehalten seien. Wie aber das Igl-Gutachten feststellte, ist eine Verkammerung, das heißt die Einrichtung einer öffentlich-rechtlichen Körperschaft mit einer Pflichtmitgliedschaft der Pflegeberufe, verfassungsmäßig möglich.

Die Mitgliedschaft in einer Berufskammer hängt nicht davon ab, ob die Pflegeberufe als freie Berufe zu verstehen sind oder ob sie vorbehaltene Tätigkeiten ausüben: „Kammermitglieder können auch Angehörige der Pflegeberufe werden, die unselbstständig (abhängig) tätig oder Beamte sind“, heißt es im Gutachten von Prof. Gerhard Igl, Kiel. Die Gesetzgebungskompetenz für die Einrichtung von Pflegekammern liege bei den Ländern, so Igl.

\section{Das Igl-Gutachten}

Igl erstellte das Rechtsgutachten im Auftrag des Deutschen Pflegerates e. V. (DPR). Ziel des Gutachtens ist es, Voraussetzungen und Anforderungen an die rechtlichen Regulierungen der Pflegeberufe und ihre Tätigkeiten zu ermitteln. Das neue Gutachten des Rechtswissenschaftlers zeigt Veränderungsbedarfe und rechtliche Grundlagen für eine Neuordnung der Pflegeberufe auf.

\section{Wer darf was im Gesundheitswe- sen machen?}

Diese Frage muss geklärt sein, um ein Kompetenzdurcheinander zu vermei-
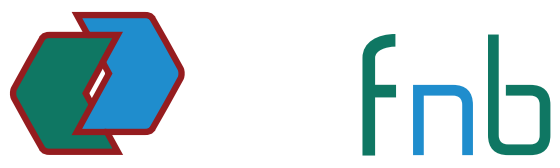

Fachverband nephrologischer Berufsgruppen

\section{Geschäftsstelle fnb}

Uschi Gasper

In den Beunen 6, 65479 Raunheim

Tel.: 06142/408549

Fax: $06142 / 408551$

E-Mail: uschi.gaspar@nephro.fachverband.de den. Daher wird die Forderung nach Vorbehaltsaufgaben - ebenso wie die nach einer Verkammerung - seit Jahren von den Pflegeverbänden vorgetragen. Leider sind auch hier die Widerstände groß. Welche Tätigkeiten im Einzelnen als Vorrang- und Vorbehaltsaufgaben zu definieren sind, richte sich nach der Qualifikation im heilkundlichen Bereich bzw. nach dem Qualifikationsvorsprung, der sich bei heilkundlichen Tätigkeiten gegenüber der ärztlichen Ausbildung ergibt.

\section{Gute Gründe für eine Kammer}

Hauptziel einer Kammer ist es, eine Berufsordnung zu definieren, die für alle Mitglieder verbindlich und verpflichtend ist. Da es in Deutschland keine Kammer für die Pflege gibt, existiert auch keine allgemeingültige Berufsordnung. Das Krankenpflegegesetz regelt zwar die Ausbildung, definiert aber nicht die Tätigkeiten und Aufgaben der Gesundheits- und Krankenpflegekräfte. Daher kann jeder Arbeitgeber für sich entscheiden, welche Aufgaben die Pflegenden übernehmen dürfen oder sollen und unter welchen Voraussetzungen dies geschieht. In einer Pflegekammer wären alle Pflegekräfte registriert. Außerdem überwacht und fördert eine Kammer die berufliche Fort- und Weiterbildung, erstellt Gutachten, benennt Sachverständige, berät den Gesetzgeber und ist am Gesetzgebungsverfahren beteiligt. Ferner trägt eine Pflegekammer dazu bei, Bildungsabschlüsse national und international zu vereinheitlichen. Bisher arbeiten in den Berufsverbänden ausschließlich ehrenamtliche Mit- arbeiter und sie haben kaum finanzielle Mittel. Würde eine Kammer mit Pflichtmitgliedschaften errichtet, könnte die Arbeit deutlich professioneller ausgerichtet werden, etwa mit einer Berufsaufsicht, Qualitätssicherung und Öffentlichkeitsarbeit. Mit der Selbstverwaltung erhielte die Pflege per Gesetz auch das Recht einer Mitgliedschaft in politischen Gremien. Derzeit werden die Mitglieder des DPR höchstens zu Expertengesprächen eingeladen und haben eine beratende Stimme. Eine Verkammerung mit Mitgliedsbeiträgen ermöglicht zudem eine finanzielle Basis, um professionelle Berufspolitik zu betreiben. Igl nannte diesen Vorteil das „institutionelle Gedächtnis“. Eine bezahlte Fachkraft kann sich den Aufgaben auf einer ganz anderen Ebene widmen, als dies ehrenamtlich Tätige können.

\section{Wie funktioniert dies bei den Nachbarn?}

In Großbritannien, Irland, Italien, Spanien oder in den USA gib es seit vielen Jahren Pflegekammern. In diesen Ländern stellen die Kliniken nur Pflegekräfte ein, die bei der Pflegekammer registriert sind und somit eine Berufslizenz haben. Die Pflegenden müssen ihre Registrierung regelmäßig erneuern und dazu eine bestimmte Anzahl an Fortbildungen nachweisen. In Deutschland können sich Pflegekräfte seit 5 Jahren freiwillig registrieren lassen. Vielleicht kann mit dieser „normativen Kraft des Faktischen“ eine Hürde auf dem Weg zur Verkammerung genommen werden. Der fnb unterstützt den DPR bei der Einrichtung von Pflegekammern. Die 1. Hürde ist es, ein Bundesland für diese Entwicklung zu gewinnen. Ist dies einmal geschafft, werden sicher andere Länder folgen. Christa Tast, Stuttgart stellvertretende Vorsitzende fnb

\section{Weitere Informationen}

Das vollständige Gutachten von Igl (weitere öffentlich-rechtliche Regulierung der Pflegeberufe und ihrer Tätigkeit) sowie Infos zu den Voraussetzungen und Anforderungen erhalten Sie über die Geschäftsstelle des Deutschen Pflegerates: www.deutscher-pflegerat.de 